\title{
Capacity Allocation in Multi-cell UMTS Networks for Different Spreading Factors with Perfect and Imperfect Power Control
}

\author{
Robert Akl \\ Dept of Computer Science and Eng. \\ University of North Texas \\ Denton, Texas, 76207 \\ rakl@cse.unt.edu
}

\author{
Son Nguyen \\ Dept of Computer Science and Eng. \\ University of North Texas \\ Denton, Texas, 76207 \\ stn@cse.unt.edu
}

\begin{abstract}
An analytical model for calculating capacity in multicell UMTS networks is presented. Capacity is maximized for different spreading factors and for perfect and imperfect power control. An analytical model is presented for approximating the user distributions in multi-cell third generation WCDMA networks using 2-dimensional Gaussian distributions by determining the means and the standard deviations of the distributions for every cell. This allows for the calculation of the inter-cell interference and the reverse-link capacity of the network. The capacity was determined for signal-to-interference threshold from $5 \mathrm{~dB}$ to $10 \mathrm{~dB}$ and spreading factor values of 256 , 64,16 , and 4 .
\end{abstract}

\section{Introduction}

3G cellular systems are identified as International Mobile Telecommunications-2000 under International Telecommunication Union and as Universal Mobile Telecommunications Systems (UMTS) by European Telecommunications Standards Institute. Besides voice capability in $2 \mathrm{G}$, the new $3 \mathrm{G}$ systems are required to have additional support on a variety of data-rate services using multiple access techniques. Code Division Multiple Access (CDMA) is the fastest-growing digital wireless technology since its first commercialization in 1994. The major markets for CDMA are North America, Latin America, and Asia (particularly Japan and Korea). In total, CDMA has been adopted by more than 100 operators across 76 countries around the globe [1].

Since the first comparisons of multiple access schemes for UMTS [2], which found that Wideband CDMA (WCDMA) was well suited for supporting variable bit rate services, several research on
WCDMA capacity has been considered. In [3], the authors present a method to calculate the WCDMA reverse link Erlang capacity based on the Lost Call Held $(\mathrm{LCH})$ model as described in [4]. This algorithm calculates the occupancy distribution and capacity of UMTS/WCDMA systems based on a system outage condition. The authors derive a closed form expression of Erlang capacity for a single type of traffic loading and compare analytical results with simulations results.

The same LCH model was also used in [5] to calculate the forward link capacity of UMTS/WCDMA systems based on the system outage condition. In the forward link, because many users share the base station (BS) transmission power, the capacity is calculated at the BS. The transmission power from the BS is provided to each user based on each user's relative need. The access in the calculation of forward link capacity is one-to-many rather than many-to-one as in the reverse link. The authors provide capacity calculation results and performance evaluation through simulation.

An alternate approach, where mobile stations (MSs) are synchronized on the uplink, i.e., signals transmitted from different MSs are time aligned at the BS, has been considered. Synchronous WCDMA looks at time synchronization for signal transmission between the BS and MS to improve network capacity. The performance of an uplink-synchronous WCDMA is analyzed in [6]. Scrambling codes are unique for each cell. MSs in the same cell share the same scrambling code, while different orthogonal channelization codes are derived from the set of Walsh codes. In [6], the potential capacity gain is about $35.8 \%$ in a multi-cell scenario with infinite number of channelization codes per cell and no soft handoff capability between MSs and BSs. However, the capacity gain in a more realistic scenario is reduced to $9.6 \%$ where soft handoff is enabled. The goal of this uplink-synchronous method in WCDMA is 
to reduce intra-cell interference. But the implementation is fairly complex while the potential capacity gain is not very high.

In this work, we will calculate the maximum reverse link capacity in UMTS/WCDMA systems for both perfect and imperfect power control with a given set of quality of service requirements and for different spreading factors.

The remainder of this paper is organized as follows. The user and interference models are presented in Section 2 . In Sections 3 and 4, we analyze capacity for perfect and imperfect power control, respectively. Spreading factors are discussed in Section 5. Numerical results are presented in Section 6, and finally Section 7 concludes the paper.

\section{User and Interference Model}

This study assumes that each user is always communicating and is power controlled by the BS that has the highest received power at the user. Let $r_{i}(x, y)$ and $r_{j}(x, y)$ be the distance from a user to BS $i$ and BS $j$, respectively. This user is power controlled by BS $j$ in the cell or region $C_{j}$ with area $A_{j}$, which BS $j$ services. This study assumes that both large scale path loss and shadow fading are compensated by the perfect power control mechanism. Let $I_{j i, g}$ be the average inter-cell interference that all users $n_{j, g}$ using services $g$ with activity factor $v_{g}$ and received signal $S_{g}$ at BS $j$ impose on BS $i$. Modifying the average inter-cell interference given by [7], it becomes

$I_{j i}^{(g)}=S_{g} v_{g} n_{j, g} \frac{e^{\left(\beta \sigma_{s}\right)^{2}}}{A_{j}} \iint_{C_{j}} \frac{r_{j}^{m}(x, y)}{r_{i}^{m}(x, y)} w(x, y) d A(x, y)$,

where $\beta=\ln (10) / 10, \sigma_{s}$ is the standard deviation of the attenuation for the shadow fading, $m$ is the path loss exponent, and $w(x, y)$ is the user distribution density at $(x, y)$. Let $\kappa_{j i, g}$ be the per-user (with service $g$ ) relative inter-cell interference factor from cell $j$ to BS $i$

$$
\kappa_{j i, g}=\frac{e^{\left(\beta \sigma_{s}\right)^{2}}}{A_{j}} \iint_{C_{j}} \frac{r_{j}^{m}(x, y)}{r_{i}^{m}(x, y)} w(x, y) d A(x, y) .
$$

The inter-cell interference density $I_{j i}^{\text {inter }}$ from cell $j$ to BS $i$ from all services $G$ becomes

$$
I_{j i}^{i n t e r}=\frac{1}{W} \sum_{g=1}^{G} I_{j i}^{(g)}
$$

where $W$ is the bandwidth of the system. Eq. (3) can be rewritten as

$$
I_{j i}^{\text {inter }}=\frac{1}{W} \sum_{g=1}^{G} S_{g} v_{g} n_{j, g} \kappa_{j i, g} .
$$

Thus, the total inter-cell interference density $I_{i}^{\text {inter }}$ from all other cells to BS $i$ is

$$
I_{i}^{\text {inter }}=\frac{1}{W} \sum_{j=1, j \neq i}^{M} \sum_{g=1}^{G} S_{g} v_{g} n_{j, g} \kappa_{j i, g}
$$

where $M$ is the total number of cells in the network.

If the user distribution density can be approximated, then, $\kappa_{j i, g}$ needs to be calculated only once. The user distribution is modeled with a 2-dimensional Gaussian function as follows [8]

$$
w(x, y)=\frac{\eta}{2 \pi \sigma_{1} \sigma_{2}} e^{-\frac{1}{2}\left(\frac{x-\mu_{1}}{\sigma_{1}}\right)^{2}} e^{-\frac{1}{2}\left(\frac{y-\mu_{2}}{\sigma_{2}}\right)^{2}},
$$

where $\eta$ is a user density normalizing parameter.

By specifying the means $\mu_{1}$ and $\mu_{2}$ and the standard deviations $\sigma_{1}$ and $\sigma_{2}$ of the distribution for every cell, an approximation can be found for a wide range of user distributions ranging from uniform to hot-spot clusters. These results are compared with simulations to determine the value of $\eta$ experimentally.

\section{WCDMA Capacity with Perfect Power Control}

In WCDMA, with perfect power control (PPC) between BSs and MSs, the energy per bit to total interference density at BS $i$ for a service $g$ is given by [9]

$$
\left(\frac{E_{b}}{I_{0}}\right)_{i, g}=\frac{\frac{S_{g}}{R_{g}}}{N_{0}+I_{i}^{\text {inter }}+I_{i}^{\text {own }}-S_{g} v_{g}}
$$

where $N_{0}$ is the thermal noise density, and $R_{g}$ is the bit rate for service $g . I_{i}^{\text {inter }}$ was calculated in section 2 . $I_{i}^{\text {own }}$ is the total intra-cell interference density caused by all users in cell $i$. Thus $I_{i}^{\text {own }}$ is given by

$$
I_{i}^{\text {own }}=\frac{1}{W} \sum_{g=1}^{G} S_{g} v_{g} n_{i, g} .
$$

Let $\tau_{g}$ be the minimum signal-to-noise ratio, which must received at a $\mathrm{BS}$ to decode the signal of a user with service $g$, and $S_{g}^{*}$ be the maximum signal power, which the user can transmit. Substituting (5) and (8) into (7), we have for every cell $i$ in the WCDMA network, the number of users $n_{i, g}$ in $\mathrm{BS} i$ for a given service 
$g$ needs to meet the following inequality constraints

$$
\tau_{g} \leq \frac{\frac{S_{g}^{*}}{R_{g}}}{N_{0}+\frac{S_{g}^{*}}{W}\left[\sum_{g=1}^{G} n_{i, g} v_{g}+\sum_{\substack{j=1, j \neq i \\ \text { for } i=1, \ldots, M .}}^{G} \sum_{g=1}^{G} n_{j, g} v_{g} \kappa_{j i, g}-v_{g}\right]},
$$

After rearranging terms, (9) can be rewritten as

$$
\begin{gathered}
\sum_{g=1}^{G} n_{i, g} v_{g}+\sum_{j=1, j \neq i}^{M} \sum_{g=1}^{G} n_{j, g} v_{g} \kappa_{j i, g}-v_{g} \leq c_{e f f}^{(g)}, \\
\quad \text { for } i=1, \ldots, M
\end{gathered}
$$

where

$$
c_{e f f}^{(g)}=\frac{W}{R_{g}}\left[\frac{1}{\tau_{g}}-\frac{R_{g}}{S_{g}^{*} / N_{0}}\right] .
$$

The maximized capacity in a WCDMA network is defined as the maximum number of simultaneous users $\left(n_{1, g}, n_{2, g}, \ldots, n_{M, g}\right)$ for all services $g=1, \ldots, G$ that satisfy (10).

\section{WCDMA Capacity with Imperfect Power Control}

The calculation of WCDMA network capacity, which was formulated in section 3, assumes perfect power control between the BSs and MSs. However, transmitted signals between BSs and MSs are subject to multi-path propagation conditions, which make the received $\left(\frac{E_{b}}{I_{o}}\right)_{i, g}$ signals vary according to a log-normal distribution with a standard deviation on the order of 1.5 to $2.5 \mathrm{~dB}$ [4]. Thus, in the imperfect power control (IPC) case, the constant value of $\left(E_{b}\right)_{i, g}$ in each cell $i$ for every user with service $g$ needs to be replaced by the variable $\left(E_{b}\right)_{i, g} \triangleq \epsilon_{i, g}\left(E_{b}\right)_{o, g}$, which is log-normally distributed. We define

$$
x_{i, g}=10 \log _{10}\left(\frac{\epsilon_{i, g}\left(E_{b}\right)_{o, g}}{I_{0}}\right),
$$

to be a normally distributed random variable with mean $m_{c}$ and standard deviation $\sigma_{c}$.

According to [4], by evaluating the $n$th moment of $\epsilon_{i, g}$ using the fact that $x_{i, g}$ is Gaussian with mean $m_{c}$ and standard deviation $\sigma_{c}$, then taking the expected value, we have

$$
E\left[\frac{\left(E_{b}\right)_{o, g}}{I_{0}} \epsilon_{i, g}\right]=\frac{\left(E_{b}\right)_{i, g}}{I_{0}} e^{\frac{\left(\beta \sigma_{c}\right)^{2}}{2}} .
$$

As a result of (13), $c_{e f f_{-} I P C}^{(g)}$ becomes $c_{e f f}^{(g)} / e^{\frac{\left(\beta \sigma c^{2}\right.}{2}}$.

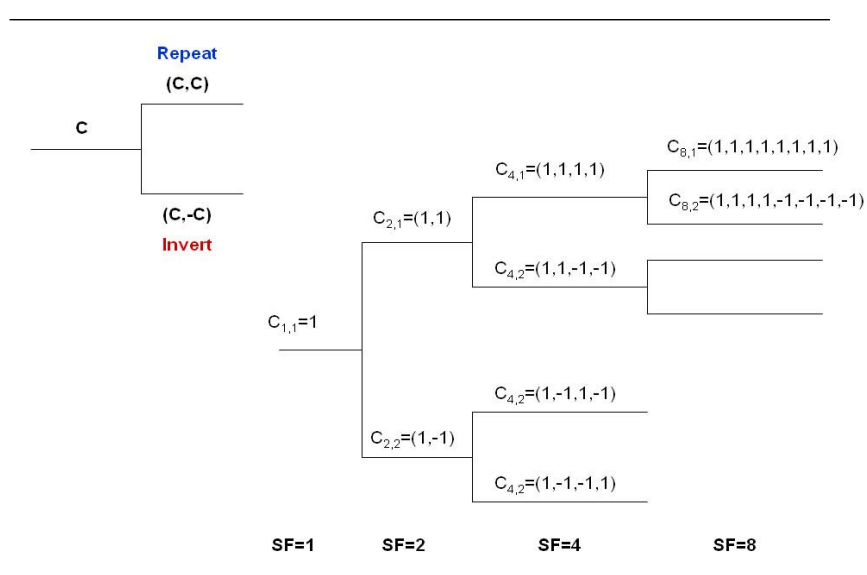

\section{Figure 1. Generation of OVSF codes for different Spreading Factors.}

\section{Spreading Factor}

Communication from a single source is separated by channelization codes, i.e. the dedicated physical channel in the uplink and the downlink connections within one sector from one MS. The Orthogonal Variable Spreading Factor (OVSF) codes, which were originally introduced in [10], were used to be channelization codes for UMTS.

The use of OVSF codes allows the orthogonality and spreading factor (SF) to be changed between different spreading codes of different lengths. Fig. 1 depicts the generation of different OVSF codes for different SF values.

\section{Numerical Results}

The results shown are for a twenty-seven cell network topology used in $[11,12]$. The COST-231 propagation model with a carrier frequency of $1800 \mathrm{MHz}$, average base station height of 30 meters and average mobile height of 1.5 meters, is used to determine the coverage region. The path loss coefficient $m$ is 4 . The shadow fading standard deviation $\sigma_{s}$ is $6 \mathrm{~dB}$. The processing gain $\frac{W}{R_{g}}$ is $6.02 \mathrm{~dB}, 12.04 \mathrm{~dB}, 18.06 \mathrm{~dB}$, and $24.08 \mathrm{~dB}$ for Spreading Factor equal to 4, 16, 64, and 256 , respectively. The activity factor, $v$, is 0.375 . Fig. 2 shows the 2-D Gaussian approximation of users uniformly distributed in the cells with $\sigma_{1}=\sigma_{2}=12000$.

The WCDMA network with 27 omnidirectional antenna cells (1 sector per cell) was analyzed for evaluation of capacity using user modeling with the 2-D Gaussian function and traditional methods of model- 


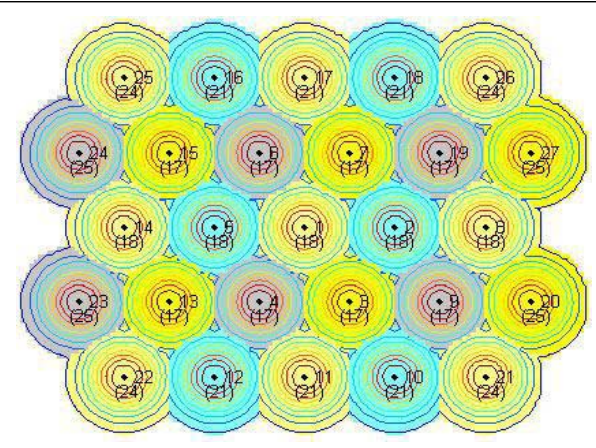

Figure 2. 2-D Gaussian approximation of users uniformly distributed in the cells. $\sigma_{1}=\sigma_{2}=$ 12000, $\mu_{1}=\mu_{2}=\mathbf{0}$.

ing uniform user distribution. The network with different values for $\frac{E_{b}}{I_{0}}$ was analyzed for different $\mathrm{SF}$ values of $4,16,64$, and 256 .

\subsection{Capacity Allocation with SF of 256}

First, we set SF to 256, which is used to carry data for the control channel. Fig. 3 shows the maximized average number of slots per sector for the 27 cells WCDMA network as the $\frac{E_{b}}{I_{0}}$ is increased from $5 \mathrm{~dB}$ to $10 \mathrm{~dB}$ and the standard deviation of imperfect power control is increased from 0 to $2.5 \mathrm{~dB}$. Because of IPC, to get the same average number of slots per sector as PPC, we have to decrease the SIR threshold by $0.5 \mathrm{~dB}$ to $1.5 \mathrm{~dB}$. Fig. 3 also shows that the traditional uniform user distribution modeling matches well with the 2-D Gaussian model.

\subsection{Capacity Allocation with SF of 64}

As a result of lowering the SF to 64 , the number of slots per sector decreases by almost a factor of 4 compared to SF equal 256 (from 60.58 to 15.56 slots when $\frac{E_{b}}{I_{o}}=7.5 \mathrm{~dB}$ in PPC) as shown in Fig. 4.

\subsection{Capacity Allocation with SF of $\mathbf{1 6}$}

As a result of lowering the SF to 16 , the number of slots per sector decreases by almost a factor of 4 compared to SF equal 64 (from 15.56 to 4.30 slots when $\frac{E_{b}}{I_{o}}$ $=7.5 \mathrm{~dB}$ in PPC) as shown in Fig. 5 .

\subsection{Capacity Allocation with SF of 4}

Next, we set SF to 4, which is used for $256 \mathrm{kbps}$ data communication between BSs and MSs. As a result of

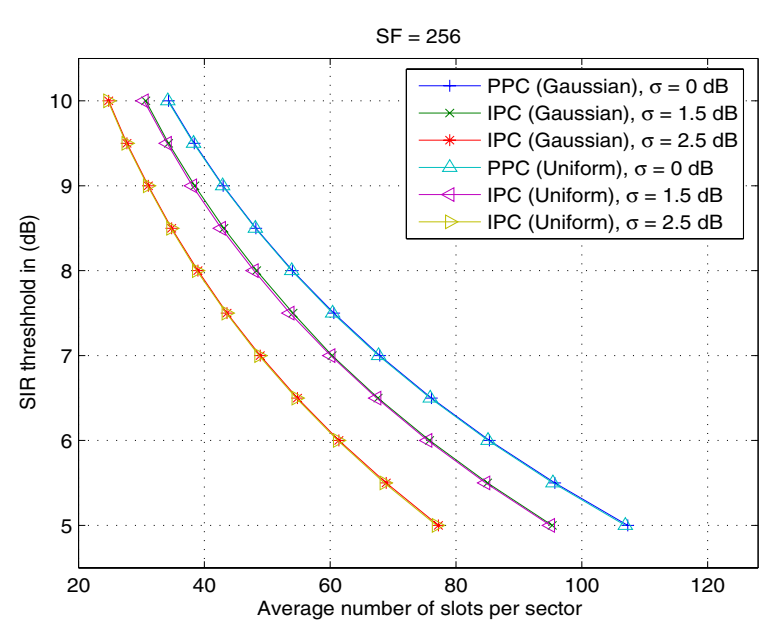

Figure 3. Average number of slot per sector for perfect and imperfect power control analysis with a Spreading Factor of 256.

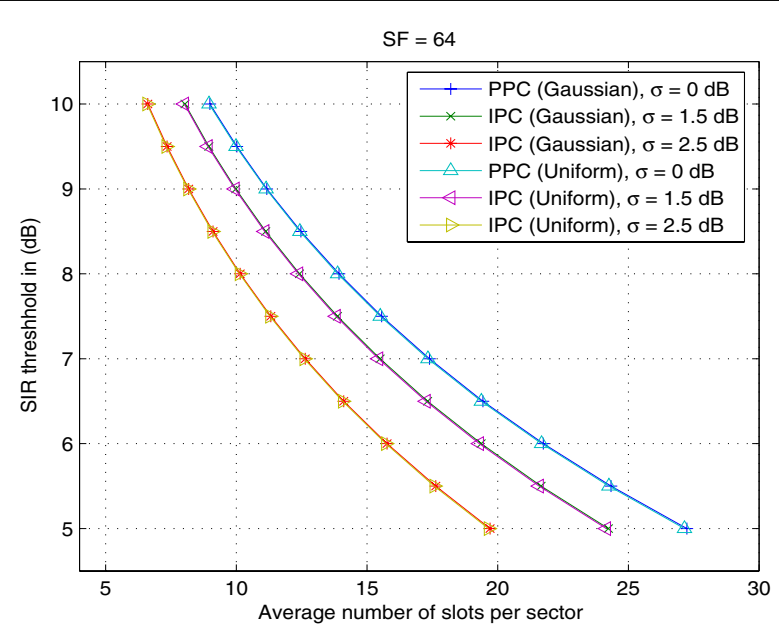

Figure 4. Average number of slot per sector for perfect and imperfect power control analysis with a Spreading Factor of 64 . 


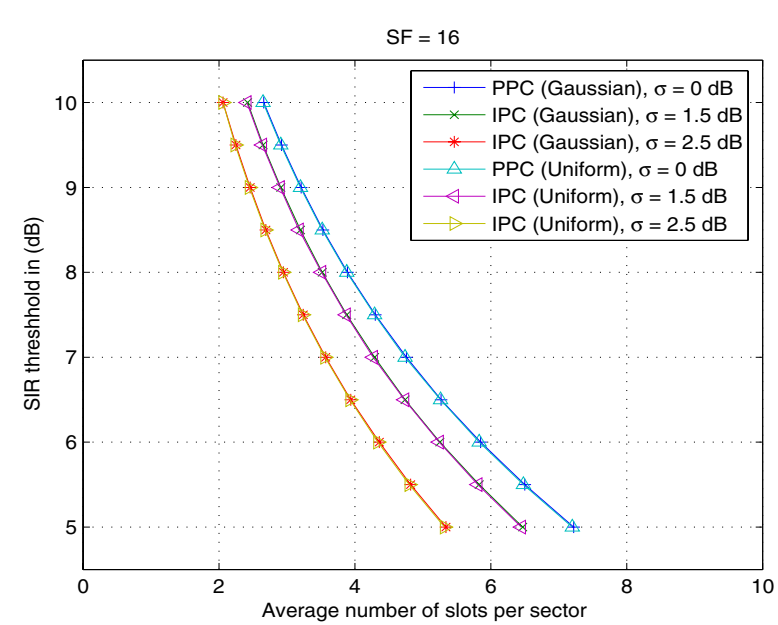

Figure 5. Average number of slot per sector for perfect and imperfect power control analysis with a Spreading Factor of 16.

lowering the SF to 4, the number of slots per sector decreases significantly to 1.49 while keeping $\frac{E_{b}}{I_{o}}=7.5 \mathrm{~dB}$ in PPC as shown in Fig. 6.

\section{Conclusions}

An analytical model has been presented for calculating capacity in multi-cell WCDMA networks. Numerical results show that the SIR threshold for the received signals is decreased by 0.5 to $1.5 \mathrm{~dB}$ due to the imperfect power control. As expected, we can have many low rate voice users or fewer data users as the data rate increases. The results also show that the determined parameters of the 2-dimensional Gaussian model matches well with traditional methods for modeling uniform user distribution. Our method of maximizing capacity is fast, accurate, and can be implemented for large multi-cell UMTS networks.

\section{References}

[1] CDMA Development Group, "CDG : Worldwide : CDMA Worldwide," http://www.cdg.org/worldwide/index.asp?h_area=0.

[2] T. Ojanpera, J. Skold, J. Castro, L. Girard, and A. Klein, "Comparison of multiple access schemes for UMTS," IEEE Veh. Technol. Conf., vol. 2, pp. 490-494, May 1997.

[3] Q. Zhang and O. Yue, "UMTS air interface voice/data capacity-part 1: reverse link analysis," IEEE Veh. Technol. Conf., vol. 4, pp. 2725 - 2729, May 2001.

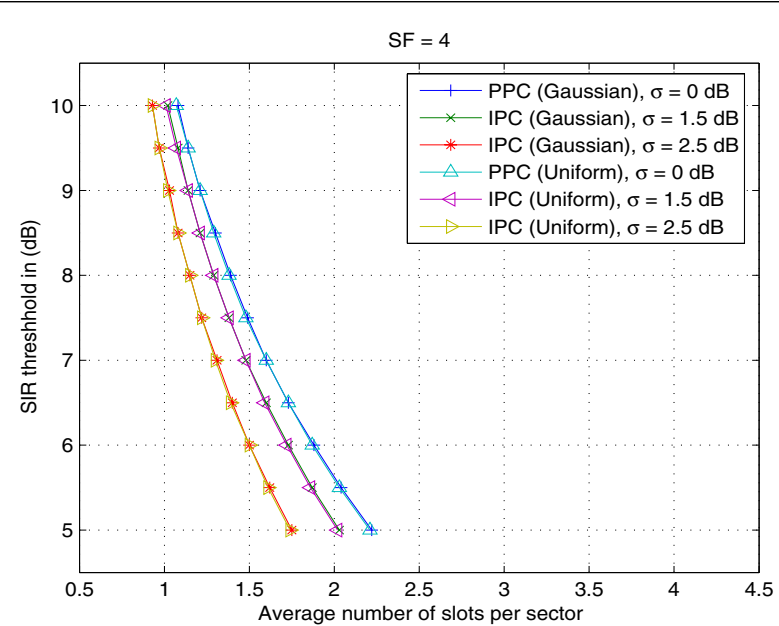

Figure 6. Average number of slot per sector for perfect and imperfect power control analysis with a Spreading Factor of 4.

[4] A. Viterbi, CDMA Principles of Spread Spectrum Communication. Addison-Wesley, 1995.

[5] Q. Zhang, "UMTS air interface voice/data capacitypart 2: forward link analysis," IEEE Veh. Technol. Conf., vol. 4, pp. 2730 - 2734, May 2001.

[6] J. Carnero, K. Pedersen, and P. Mogensen, "Capacity gain of an uplink-synchronous WCDMA system under channelization code constraints," IEEE Veh. Technol. Conf., vol. 53, pp. 982 - 991, July 2004.

[7] R. Akl, M. Hegde, M. Naraghi-Pour, and P. Min, "Multicell CDMA network design," IEEE Trans. Veh. Technol., vol. 50, no. 3, pp. 711-722, May 2001.

[8] S. Nguyen and R. Akl, "Approximating user distributions in WCDMA networks using 2-D Gaussian," International Conf. on Comput., Commun., and Control Technol., July 2005, accepted.

[9] D. Staehle, K. Leibnitz, K. Heck, B. Schroder, A. Weller, and P. Tran-Gia, "Approximating the othercell interference distribution in inhomogenous UMTS networks," IEEE Veh. Technol. Conf., vol. 4, pp. 1640-1644, May 2002.

[10] A. F., S. M., and O. K., "Tree-structured generation of orthogonal spreading codes with different lengths for forward link of DS-CDMA mobile radio," Electr. Lett., vol. 33, pp. 27-28, January 1997.

[11] R. Akl and A. Parvez, "Impact of interference model on capacity in CDMA cellular networks," Proceedings of SCI 04: Communication and Network Systems, Technologies and Applications, vol. 3, pp. 404-408, July 2004.

[12] R. Akl, M. Hegde, and M. Naraghi-Pour, "Mobilitybased CAC algorithm for arbitrary traffic distribution in CDMA cellular systems," IEEE Trans. Veh. Technol., vol. 54, pp. 639-651, March 2005. 